\begin{tabular}{|c|c|c|}
\hline \multirow{3}{*}{$\begin{array}{r}\text { Case Reports in } \\
\text { Gastroenterology }\end{array}$} & \multirow{2}{*}{\multicolumn{2}{|c|}{ Case Rep Gastroenterol 2013;7:30-36 }} \\
\hline & & \\
\hline & $\begin{array}{l}\text { DOI: } 10.1159 / 000346693 \\
\text { Published online: January 23, } 2013\end{array}$ & $\begin{array}{l}\text { ( ) } 2013 \text { S. Karger AG, Basel } \\
1662-0631 / 13 / 0071-0030 \$ 38.00 / 0 \\
\text { www.karger.com/crg }\end{array}$ \\
\hline
\end{tabular}

\title{
Microinvasion of High-Grade Pancreatic Intraepithelial Neoplasia
}

\author{
Natsuko Kawada ${ }^{a}$ Hiroyuki Uehara ${ }^{a}$ Ryoji Takada ${ }^{a}$ Takuo Yamai ${ }^{a}$ \\ Nobuyasu Fukutake $^{a} \quad K^{2}$ azuhiro Katayama ${ }^{a}$ Akemi Takenaka $^{\mathrm{b}}$ \\ Shigenori Nagata ${ }^{b}$ Yasuhiko Tomita ${ }^{b}$ \\ Departments of a Gastroenterology and ${ }^{b}$ Pathology, Osaka Medical Center for Cancer and \\ Cardiovascular Diseases, Osaka, Japan
}

\section{Key Words}

PanIN-3 · Microinvasion · Precursor lesion

\begin{abstract}
High-grade pancreatic intraepithelial neoplasia (PanIN-3) is recognized as a precursor lesion of invasive ductal carcinoma (IDC). However, histological evidence that PanIN-3 invades beyond the basement membrane of pancreatic ductal epithelium, that is, the moment PanIN-3 becomes IDC, has not been captured yet. This may be because PanINs which are microscopic papillary or flat lesion rarely develop clinical symptoms and are not detectable on imaging examination. On the other hand, most IDCs were found in the advanced stage with massive invasion. In this report, PanIN-3 obstructed several branch pancreatic ducts and subsequently caused pancreatitis which developed clinical symptom and was detectable as a pancreatic mass in imaging studies. A 65-year-old woman was referred to our institution for further examination of her repeated pancreatitis. Abdominal ultrasound revealed a low echoic mass of $13 \mathrm{~mm}$ in diameter in the pancreatic body without upstream dilatation of the main pancreatic duct (MPD). Endoscopic retrograde pancreatography showed a strictured segment of $2 \mathrm{~mm}$ in length in the MPD at the pancreatic body. Cytological examination of pancreatic juice revealed adenocarcinoma and distal pancreatectomy was performed. A resected specimen revealed a whitish mass of $15 \mathrm{~mm}$ in diameter in the pancreatic body, which was identified as pancreatitis by histological examination. Papillary growth of PanIN-3 was seen mainly in the branch ducts. Each PanIN-3 was located separately in the branch ducts with normal epithelia in the MPD between them. In three adjacent branch ducts, PanIN-3 was observed to be invading microscopically beyond the basement membrane.
\end{abstract}




\section{Introduction}

Pancreatic intraepithelial neoplasia (PanIN) is a nomenclature for microscopic proliferative epithelial lesions of the pancreas which was proposed in 2001 by Hruban et al. [1]. PanIN is assumed to be a precursor lesion of invasive ductal carcinoma (IDC). Precursor lesions of IDC also include intraductal papillary mucinous neoplasm (IPMN) and mucinous cystic neoplasm. PanIN is divided into three grades, PanIN-1, -2 , and -3 , according to the cytological and architectural atypia. PanIN-1 is further subdivided into flat (PanIN-1A) and papillary types (PanIN-1B).

Histologically, PanIN has been considered to progress toward IDC. This hypothesis has arisen from the observation of the resected specimens, in which synchronous or metachronous occurrence of PanIN and IDC were seen. PanIN had been reported to be more common in the pancreas with IDC than without [2,3]. It has also been reported that IDC occurred in the remnant pancreas several years after partial pancreatectomy for high-grade PanIN had been performed $[4,5]$.

Several studies have demonstrated higher frequencies of genetic alterations such as K-ras mutation or DPC4 inactivation in higher-grade PanIN lesions [6-9]. Thus, it is hypothesized that pancreatic ductal lesions may progress from a histologically normal duct through flat lesion (PanIN-1A), papillary lesion (PanIN-1B), atypical lesion (PanIN-2) to severely atypical lesion (PanIN-3). PanIN-3 is likely to develop eventually into IDC because PanIN-3 and IDC share critical genetic abnormalities. However, the histological evidence that PanIN-3 invades beyond the basement membrane of pancreatic ductal epithelium, that is, the moment PanIN-3 becomes IDC, has not been captured yet.

As defined by the consensus guideline [10], PanIN is a microscopic papillary or flat, noninvasive epithelial neoplasia that is usually $<5 \mathrm{~mm}$ in diameter. Generally, PanINs rarely develop clinical symptoms and are not detectable on imaging examination. Therefore, the clinical entity of PanIN-3 has not been established.

In this report, PanIN-3 obstructed several branch pancreatic ducts and subsequently caused pancreatitis which developed clinical symptom and was detectable as a pancreatic mass on imaging studies. Since pancreatic cancer was suspected, further examinations were performed. Pancreatic juice cytology revealed adenocarcinoma, so the patient underwent surgery. Histological examination of the resected specimens showed PanIN-3 slightly invading beyond the basement membrane of the ductal epithelia accompanied by fibrotic changes caused by occlusion of branch ducts.

\section{Case Report}

A 65-year-old woman had been admitted to a former hospital for acute pancreatitis, which recurred 2 months thereafter. She was referred to our center for further examination of her pancreas after the second pancreatitis had been relieved. She was slightly thin, showing a body mass index of 20.2, and had neither smoking nor drinking habits. She had no family history of pancreatic diseases. Laboratory data showed no abnormalities including serum levels of amylase and tumor markers related to pancreatic diseases. Abdominal ultrasound revealed a low echoic mass of $13 \mathrm{~mm}$ in diameter in the pancreatic body without upstream dilatation of the main pancreatic duct (MPD) (fig. 1a, b). Contrast enhanced computed tomography showed neither pancreatic mass nor dilated MPD (fig. 1c, d). Endoscopic ultrasound showed a low echoic mass of $20 \mathrm{~mm}$ in diameter in the pancreatic body (fig. 1e). Since pancreatic cancer was suspected in the pancreatic body, she underwent endoscopic 
retrograde pancreatography, which showed a strictured segment of $2 \mathrm{~mm}$ in length in the MPD at the pancreatic body (fig. 1f). Cytological examination of the pancreatic juice obtained during endoscopic retrograde pancreatography revealed adenocarcinoma (fig. 1g). The patient was diagnosed with pancreatic body cancer without obvious vascular involvement (T3, N0, M0, stage IIA, according to the UICC classification) and underwent distal pancreatectomy.

The resected specimens were fixed in formalin and cut at a slice thickness of $5 \mathrm{~mm}$ as shown in fig. 2a. PanIN lesions were observed in 15 slices (fig. 2b). A slice in the pancreatic body (slice no. 5) showed a whitish mass of $15 \mathrm{~mm}$ in diameter (fig. 2c) which was identified as pancreatitis by subsequent microscopic examination (fig. 2d). Histological examination revealed papillary growth of intraductal epithelia with significant nuclear atypia which was classified as PanIN-3 according to the definition of the consensus guideline [10] mainly in the branch ducts (fig. 2b, e, g). PanIN-2 and -1 were also seen around PanIN-3 (fig. 2b, e, f). PanIN-3s were located separately in branch ducts with normal epithelia in the MPD between them (fig. 2b, h, i), indicating that they originated from different sites of the branch ducts. PanIN-3s invaded microscopically beyond the basement membrane in three adjacent branch ducts (fig. 2j). Histologically, this case was diagnosed as IDC of the pancreatic body (T1, N0, M0, stage I). The patient was doing well without any signs of recurrence 40 months after surgery.

\section{Discussion}

High-grade PanIN (PanIN-3) is recognized as a best-defined precursor lesion of IDC based on genetic as well as histological observations. PanIN-3 has been reported to share some genetic alterations with IDC [6-9] and to be found usually in the pancreas with IDC [25]. However, the direct evidence of the moment when PanIN-3 invades beyond the basement membrane to become IDC has not been captured yet. This is the first case report in which microinvasion of PanIN-3 was histologically confirmed in the resected pancreatic specimens.

Considerable ambiguities had existed in the distinction between PanIN and IPMN, therefore the definitions of these two lesions were revised at the consensus meeting held at Johns Hopkins Hospital in 2003 [10]. According to the revised definitions, PanIN is a microscopic, papillary or flat epithelial neoplasm arising in the pancreatic duct and differs from IPMN, which is defined as a grossly visible, mucin-producing, predominantly papillary epithelial neoplasm. In this case, pancreatic epithelial neoplasm itself was not identified in any radiological examinations, and mucin production was not evident in pancreatic ductal epithelia by microscopic observation. Thus, this case was diagnosed as PanIN.

A few reports had suggested that minimally invasive carcinoma should be distinguished from invasive IPMN because this subgroup of IPMN showed apparently better prognosis [11-13]. However, the precise definition of minimally invasive carcinoma has not been established $[14,15]$ and has been recently proposed by some studies $[16,17]$. In this case, the patient has been alive without any signs of recurrence for more than 3 years. Since there has been no concept of minimally invasive PanIN to date, this case is classified as IDC in spite of excellent outcome. A new notion that minimally invasive PanIN should be distinguished from IDC might be widely accepted in the future.

In this case, PanIN-3 lesions were located separately in several branch ducts without involving the epithelia of the main duct between them. This fact indicates that PanIN does not arise from one small portion of pancreatic ductal epithelium and spread creeping along the epithelia, but arises multicentric. It is well known that IPMN, another precursor lesion of 
IDC, occurs multicentric. As the whole pancreas may be involved in the genetic alteration which is related to carcinogenesis, it is not difficult to understand that PanIN occurs independently in different sites of the pancreas.

Several cases dealing with multiple occurrence of IDC have been reported as rare clinical manifestation $[18,19]$. Interestingly in our case, microinvasion was observed synchronously in three different branch ducts. This observation could explain the rapid growth of pancreatic ductal carcinoma as follows: microinvasion initially occurs in several pancreatic ducts; each invasion independently progresses and eventually makes a fusion mass. This observation could also explain the multiple occurrence of IDC when microinvasions occur independently distinct from each other.

Unlike IPMN, PanIN-3 is hardly detected in clinical practice because PanIN-3 itself is not visible on imaging examination. In this case, PanIN-3 was successfully detected due to pancreatitis caused by the intraductal growth of PanIN-3 and subsequent occlusion of the pancreatic ducts. Pancreatitis might be a candidate for the diagnostic clues to the early detection of pancreatic ductal carcinoma within the preinvasive stage. This proposal is consistent with previous observations [20]. There are other known diagnostic clues to early detection, such as pancreatic cysts or dilatation of the MPD [21]. In this case, however, neither pancreatic cyst nor dilatation of the MPD was observed.

The detection of pancreatic ductal carcinoma in the preinvasive stage is the most promising way of improving patient survival. We hope that our report will contribute to the further understanding of early development of pancreatic ductal carcinoma.

\section{References}

-1 Hruban RH, Adsay NV, Albores-Saavedea J, et al: Pancreatic intraepithelial neoplasia: a new nomenclature and classification system for pancreatic ductal lesions. Am J Surg Pathol 2001;25:579-586.

-2 Andea A, Sarkar F, Adsay NV: Clinicopathological correlates of pancreatic intraepithelial neoplasia: a comparative analysis of 82 cases with and 152 cases without pancreatic ductal adenocarcinoma. Mod Pathol 2003;16:996-1006.

3 Recavarren C, Labow DM, Liang J, et al: Histologic characteristics of pancreatic intraepithelial neoplasia associated with different pancreatic lesions. Hum Pathol 2011;42:18-24.

4 Takaori K, Kobayashi Y, Matsusue S, et al: Clinicopathological features of pancreatic intraepithelial neoplasias and their relationship to intraductal papillary-mucinous tumors. J Hepatobiliary Pancreat Surg 2003;10:125-136.

5 McCarthy DM, Brat DJ, Wilentz RE, et al: Pancreatic intraepithelial neoplasia and infiltrating adenocarcinoma: analysis of progression and recurrence by DPC4 immunohistochemical labeling. Hum Pathol 2001;32:638-642.

-6 Wilentz RE, Iacobuzio-Donahue C, Argani P, et al: Loss of expression of Dpc4 in pancreatic intraepithelial neoplasia: evidence that DPC4 inactivation occurs late in neoplastic progression. Cancer Res 2000;60:20022006.

7 Fukushima N, Sato N, Ueki T, et al: Aberrant methylation of preproenkephalin and p16 genes in pancreatic intraepithelial neoplasia and pancreatic ductal adenocarcinoma. Am J Pathol 2002;160:1573-1581.

-8 Sato N, Fukushima N, Hruban RH, et al: CpG island methylation profile of pancreatic intraepithelial neoplasia. Mod Pathol 2008;21:238-244.

-9 Lohr M, Kloeppel G, Maisonneuve P, et al: Frequency of K-ras mutations in pancreatic intraductal neoplasia associated with pancreatic ductal adenocarcinoma and chronic pancreatitis: a metaanalysis. Neoplasia 2005;7:17-23.

10 Hruban RH, Takaori K, Klimstran DS, et al: An illustrated consensus on the classification of pancreatic intraepithelial neoplasia (PanIN) and intraductal papillary mucinous neoplasms (IPMNs). Am J Surg Pathol 2004;28:977-987.

11 Suzuki Y, Atomi Y, Sugiyama M: Cystic neoplasm of the pancreas: a Japanese multi-institutional study of intraductal papillary mucinous tumor and mucinous cystic tumor. Pancreas 2004;28:241-246.

12 Nakagohri T, Asano T, Kenmochi T, et al: Long-term surgical outcome of noninvasive and minimally invasive intraductal papillary mucinous adenocarcinoma of the pancreas. World J Surg 2002;26:1166-1169.

13 Yamao K, Ohashi K, Nakamura T, et al: The prognosis of intraductal papillary mucinous tumors of the pancreas. Hepatogastroenterology 2000;47:1129-1134. 
Kawada et al.: Microinvasion of High-Grade Pancreatic Intraepithelial Neoplasia

14 International Agency for Research on Cancer: WHO Classification of Tumours of the Digestive System, ed 4. Lyon, 2010.

15 Japan Pancreas Society: Classification of Pancreatic Cancer, ed 6. Tokyo, Kanehara, 2003.

16 Nara S, Shimada K, Kosuge T, et al: Minimally invasive intraductal papillary-mucinous carcinoma of pancreas: clinicopathologic study of 104 intraductal papillary-mucinous neoplasms. Am J Surg Pathol 2008;32:243-255.

17 Kim J, Jang KT, Park SM, et al: Prognostic relevance of pathologic subtypes and minimal invasion in intraductal papillary mucinous neoplasms of the pancreas. Tumour Biol 2011;32:535-542.

18 Naito Y, Eriguchi N, Kume T, et al: Multi-centric pancreatic cancer without PanIN lesion. J Hepatobiliary Pancreat Surg 2009;16:699-703.

19 Izumi S, Nakamura S, Mano S, et al: Resection of four synchronous invasive ductal carcinomas in the pancreas head and body associated with pancreatic intraepithelial neoplasia: report of a case. Surg Today 2009;39:1091-1097.

20 Takaori K, Matsusue S, Fujikawa T, et al: Carcinoma in situ of the pancreas associated with localized fibrosis: a clue to early detection of neoplastic lesions arising from pancreatic ducts. Pancreas 1998;17:102-105.

21 Tanaka S, Nakao M, Ioka T, et al: Slight dilatation of the main pancreatic duct and presence of pancreatic cysts as predictive signs of pancreatic cancer: a prospective study. Radiology 2010;254:965-972. 

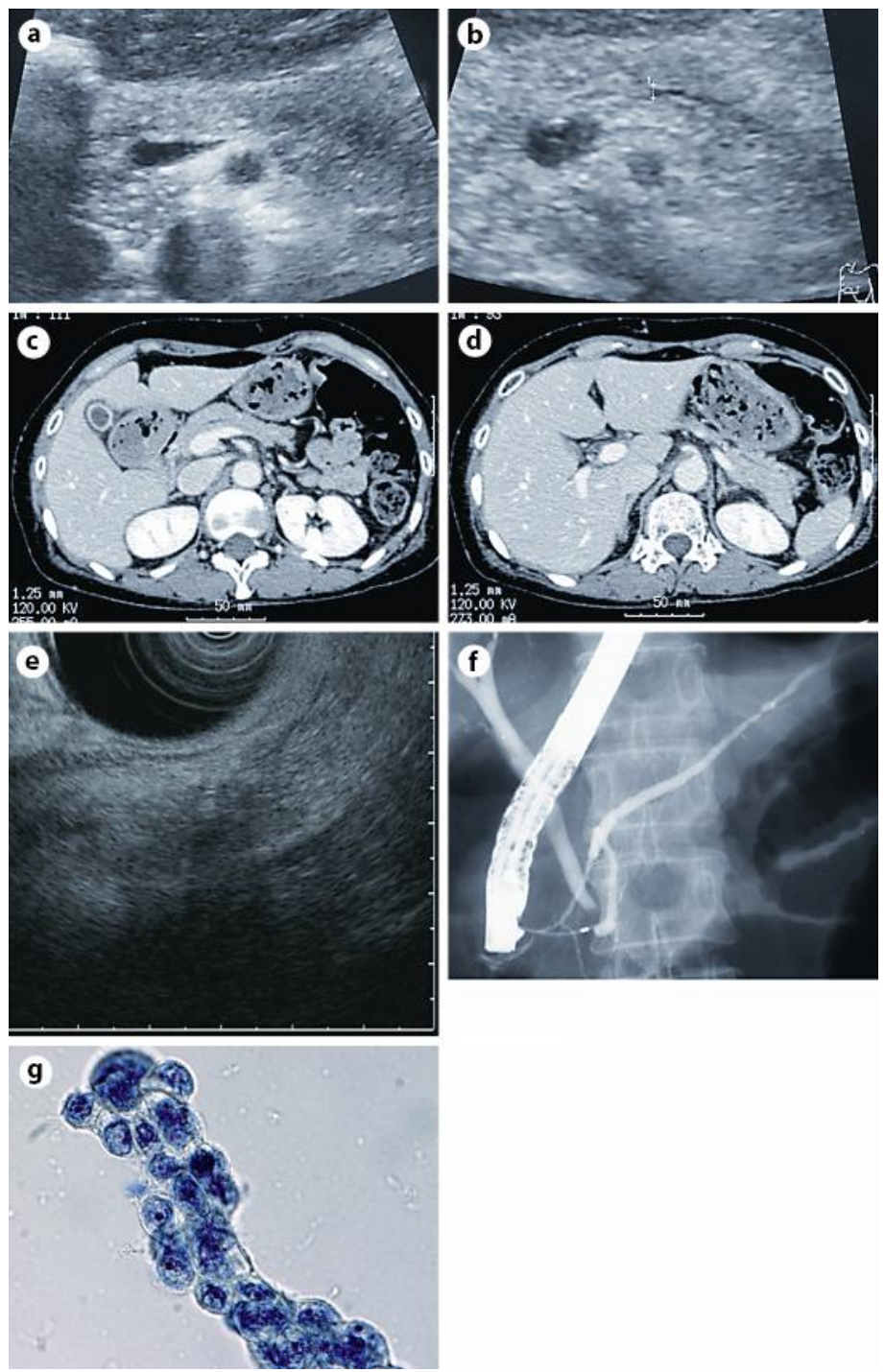

Fig. 1. a, b Abdominal ultrasound revealed a low echoic mass of $13 \mathrm{~mm}$ in diameter in the pancreatic body. Dilatation of the MPD was not observed. $\mathbf{c}$, $\mathbf{d}$ Contrast enhanced computed tomography showed neither a pancreatic mass nor a dilated MPD. e Endoscopic ultrasound showed a low echoic mass of $20 \mathrm{~mm}$ in diameter in the pancreatic body. $\mathbf{f}$ Endoscopic retrograde pancreatography revealed a strictured segment of $2 \mathrm{~mm}$ in length in the MPD without obvious upstream dilatation at the pancreatic body. $\mathrm{g}$ Pancreatic juice cytology revealed adenocarcinoma (Papanicolaou stain). 


\section{Case Reports in \\ Gastroenterology}
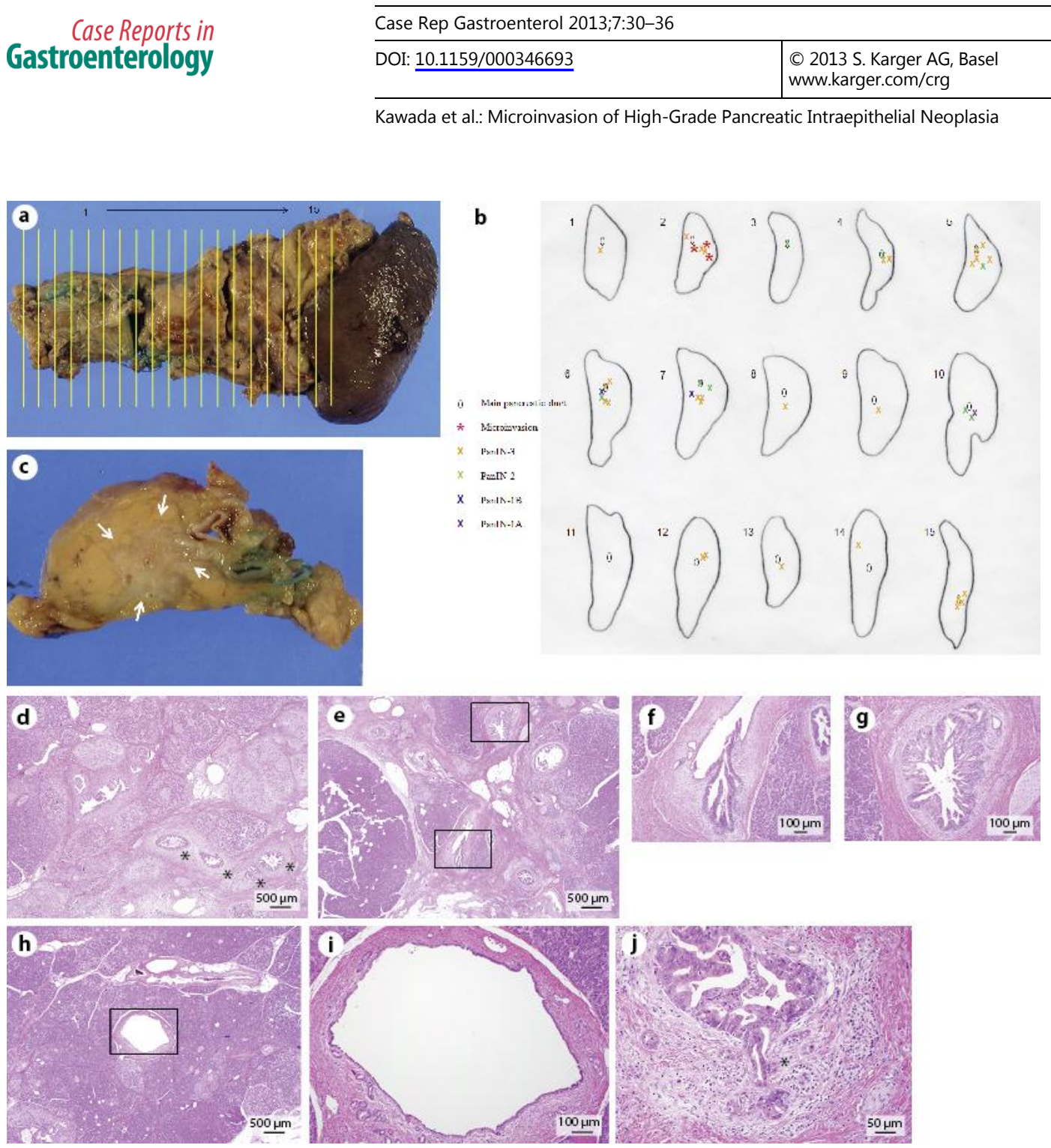

Fig. 2. a The resected specimens were cut at a slice thickness of $5 \mathrm{~mm}$. PanIN lesions were observed in 15 slices. b Schematic drawing of the localization of PanIN lesions. PanIN-3s were observed mainly in the branch pancreatic ducts accompanied by normal epithelia in the MPDs between them. PanIN-2 and -1 were also seen around PanIN-3. Microinvasions of PanIN-3 were observed in three different branch pancreatic ducts. c A whitish mass of $15 \mathrm{~mm}$ in diameter was observed in the pancreatic body (arrows) and corresponded to pancreatitis shown in $\mathbf{d}$. $\mathbf{d}$ Pancreatitis caused by occlusion of the branch pancreatic ducts (H\&E). Fibrotic change of acinus was seen around the branch ducts that were almost occluded by the papillary growth of PanIN-3 (asterisks). e-g PanIN-3 (g) and PanIN-2 (f) lesions observed in the MPD and branch ducts (H\&E, overview in e and detailed view of the black boxes in $\mathbf{f}$ and $\mathbf{g}$ ). $\mathbf{h}$, i Normal epithelia in the MPD observed between PanIN lesions in the branch ducts (H\&E, slice no. 8, overview in $\mathbf{h}$ and detailed view of the black box in i). $\mathbf{j}$ Microinvasion of PanIN-3 (H\&E). PanIN-3 slightly invaded beyond the basement membrane of intraductal epithelia (asterisk). 